\title{
A CHARACTERIZATION OF MINKOWSKIAN GEOMETRY
}

\section{NORMAN DAVIDS}

Menger called a finitely-compact metric space in which any two distinct points are contained in exactly one set congruent to a euclidean straight line a straight-line space (S.L. space) [1]. ${ }^{1} \mathrm{He}$ observed that in such spaces a segment (or vector) addition can be defined in a very simple way (see $\$ 2$ of the present paper) which satisfies all requirements for an abelian group except, in general, the associative law. Menger then put the question of determining those geometries in which the associative law holds. It is the purpose of the present note to show that the Minkowskian geometries furnish the answer. ${ }^{2}$

1. Straight-line spaces. ${ }^{3}$ By a straight-line (S.L.) in a metric space $R$ is understood a continuous curve which may be mapped congruently onto the real axis, that is, which admits of a parametrization $P(\tau),-\infty<\tau<\infty$, with $P\left(\tau_{1}\right) P\left(\tau_{2}\right)=\left|\tau_{1}-\tau_{2}\right|$. The numbers $\tau$ are the isometric coordinates of the points of the S.L. A segment joining two points $P\left(\tau_{1}\right), Q\left(\tau_{2}\right) \subset R$, written $(P Q)$, is a congruent image of the closed interval between $\tau_{1}$ and $\tau_{2}$.

An S.L. space is one in which any pair of its points $X, Y$ is contained in a unique S.L. $[X Y]$. The following conditions are sufficient for an S.L. space: the space is (1) metric, (2) finitely-compact, (3) convex, (4) externally convex, (5) if $\left(X Y Z_{1}\right),\left(X Y Z_{2}\right)$, and $Y Z_{1}=Y Z_{2}$, then $Z_{1}=Z_{2}$. We shall suppose, hereafter, that these conditions hold.

If $X_{n} \rightarrow X, Y_{n} \rightarrow Y$ and $X \neq Y$ then $\left[X_{n} Y_{n}\right] \rightarrow[X Y]$.

According to Menger $M$ is an internal center of $X$ and $Y$, written $M(X, Y)$, if $(X M Y)$ and $X M=M Y$. Also, if $(E X Y)$ and $E X=X Y$, $E$ is an external center of $X$ and $Y$, written $E(X, Y)$ or $(Y, X) E$. In an S.L. space every point-pair has a unique internal center and two external centers.

2. Addition of segments in S.L. spaces. Let $(O X),(O Y)$ be two arbitrary segments in $R$ having the end point $O$ in common. We shall call the segment $(O P)$ a sum of the two given segments, written

Received by the editors May 27, 1946.

${ }^{1}$ Numbers in brackets refer to the references cited at the end of the paper.

2 The above problem, together with a conjecture as to the answer, was suggested by $\mathrm{H}$. Busemann.

${ }^{3}$ For explanations of the notations used here, as well as proofs of the statements of this section, see Busemann [2, chaps. I and III]. 
$(O P)=(O X)+(O Y)$, if $M(O, P)=M(X, Y)$. Since $O$ will remain fixed throughout, we shall write simply $P=X+Y$. This relation has the following properties:

1. There exists exactly one sum $P=X+Y$. For if $M(X, Y)$, and $P=(O, M) E$, then $M=M(O, P)$, so that the sum exists. On the other hand if $P^{\prime}=X+Y$, then $M\left(O, P^{\prime}\right)=M(X, Y)=M(O, P)$, hence $P^{\prime}=(O, M) E$, so that, by the convexity of the space, $P^{\prime}=P$.

2. Addition is commutative. For $M(X, Y)=M(Y, X)$.

3. If $O, X, Y$ are contained in the same S.L., then $X\left(\tau_{1}\right)+Y\left(\tau_{2}\right)$ $=P\left(\tau_{1}+\tau_{2}\right)$, that is, the isometric coordinates of the sum are the sum of the isometric coordinates.

4. $P=X+Y$ is continuous in $X$ and $Y$. For $M(X, Y)$ is continuous in $X$ and $Y$ and $P=(O, M) E$ is continuous in $M$.

5. $O+X=X$.

6. $M(O, X)+M(O, X)=X$, and we may write $M=X / 2$.

7. There exists exactly one point $Y$ such that $X+Y=P$. For if $M=M(O, P)$ and $Y=(X, M) E$, then $Y$ is the (unique) required point, and we may write $Y=P-X$.

8. We shall write $O-Y$ as $-Y . Y+(-Y)=O$.

The set of segments through $O$ satisfy all the group axioms for addition except the associative law. ${ }^{4}$ The following is an example where it fails to hold: Consider the representation of the hyperbolic $(h)$ plane inside the euclidean (e) unit circle $K$ in which the $h$.S.L. are the segments of the $e$.S.L. inside $K$. Let $g, h$ be two S.L. through the center $O$ and $X \subset g, Y \subset h$ two points $h$-equidistant from $O$ (hence also $e$-equidistant from $O)$. The $h$ mid-point $M$ of $(X Y)$ is also the $e$ mid-point. Calling $Z=X+Y$ in the sense of the $h$-metric, we have $e(M Z)$ $<e(O M)$, where $e(\quad)$ denotes the $e$-distance. Hence the intersection $P=(Z(-Y)) \cdot(O X)$ is such that $e(O P)<e(P X)$, hence $h(O P) \neq h(P X)$, so that $P$ cannot be the $h$ mid-point of $(O X)$. Thus

$$
X+[Y+(-Y)]=X ; \quad(X+Y)+(-Y)=Z+(-Y) \neq X .
$$

3. The main theorem. An S.L. space $R$ has associative segment-addition for every triple of segments around a point if and only if its geometry is Minkowskian.

We show first that any two S.L. through $O$ are contained in a twodimensional Minkowski space.

We shall suppose an S.L. space $R$ in which the associative law of segment-addition holds around a point $O$.

I. Let $g$ and $g^{\prime}$ (hereinafter fixed in the discussion) be two arbitrary S.L. through $O$. The totality of points $Z \subset R$ such that $X+Y=Z$,

\footnotetext{
${ }^{4}$ Such systems, called loops, have been studied by Albert. See [3].
} 
$X \subset g, Y \subset g^{\prime}$ will be called the plane $p$ determined by $g$ and $g^{\prime}$, written $p\left(g, g^{\prime}\right)$. $p$ contains $g$ and $g^{\prime}$.

II. The sum and difference of two points $Z_{1} \subset p, Z_{2} \subset p$ is also in $p$. For $Z_{1}=X_{1}+Y_{1}, Z_{2}=X_{2}+Y_{2}, X_{1}, X_{2}<g, Y_{1}, Y_{2} \subset g^{\prime}$ and $Z_{1}+Z_{2}$ $=\left(X_{1}+Y_{1}\right)+\left(X_{2}+Y_{2}\right)=\left(X_{1}+X_{2}\right)+\left(Y_{1}+Y_{2}\right)$. Since $X_{1}+X_{2} \subset g$ and $Y_{1}+Y_{2} \subset g^{\prime}, Z_{1}+Z_{2} \subset p$.

III. If $Z_{1}, Z_{2} \subset p$ then $M\left(Z_{1}, Z_{2}\right), E\left(Z_{1}, Z_{2}\right)$ and $\left(Z_{1}, Z_{2}\right) E$ are also in $p$. First we note that if $Z=X+Y$, then $M(O, Z)=X / 2+Y / 2$, since $2(X / 2+Y / 2)=(X / 2+Y / 2)+(X / 2+Y / 2)=(X / 2+X / 2)$ $+(Y / 2+Y / 2)=X+Y=Z$. We may thus write $M(O, Z)=Z / 2$. Using this notation we have

$$
\begin{gathered}
M\left(Z_{1}, Z_{2}\right)=Z_{1} / 2+Z_{2} / 2, \quad E\left(Z_{1}, Z_{2}\right)=2 Z_{1}-Z_{2}, \\
\left(Z_{1}, Z_{2}\right) E=2 Z_{2}-Z_{1} .
\end{gathered}
$$

In the same way we can show that $m Z / n=m X / n+m Y / n$. Finally since $Z$ is continuous in $X$ and $Y$, letting $m / n \rightarrow k$, we have $k Z=k X$ $+k Y$, where $k$ is any real number.

IV. $A$ plane $p$ is finitely-compact. Suppose a bounded set $\{Z\}$ in $p$. Since $R$ is finitely-compact there exists a point $Z \subset R$ and a sequence $Z_{n} \subset\{Z\}$ such that $Z_{n} \rightarrow Z$. Let $Z_{n}=X_{n}+Y_{n}$. It must be shown that $X_{n}$ and $Y_{n}$ are bounded. Otherwise subsequences, which we again denote by $X_{n}, Y_{n}$, would tend to infinity. Let $P \subset g(P \neq 0)$ be a fixed point and let $k=O X_{n} / O P$. Then

$$
Z_{n}^{\prime}=Z_{n} / k=X_{n} / k+Y_{n} / k=P+Y_{n} / k,
$$

or $Z_{n}^{\prime}-Y_{n} / k=P$. Since $k \rightarrow \infty, Z_{n}^{\prime} \rightarrow 0$, and since $Y_{n} / k \subset g^{\prime}$, their difference cannot be a point of $g$, for $n$ sufficiently large. Thus, for some subsequence, since $R$ is finitely-compact, $X_{n} \rightarrow X, Y_{n} \rightarrow Y$, so that $Z=X+Y$, and hence $Z \subset p$.

V. Every point $Z \subset p$ is uniquely represented as a sum $Z=X+Y$, $X \subset g, Y \subset g^{\prime}$. For if $Z=X_{1}+Y_{1}=X_{2}+Y_{2}, X_{1}, X_{2} \subset g, Y_{1}, Y_{2} \subset g^{\prime}$, then $Z-Y_{1}=\left(X+Y_{1}\right)-Y_{1}=\left(X_{2}+Y_{2}\right)-Y_{1}$ which becomes, by the associative law, $X_{1}+\left(Y_{1}-Y_{1}\right)=X_{2}+\left(Y_{2}-Y_{1}\right)$, or $X_{1}=X_{2}+\left(Y_{2}-Y_{1}\right)$. Since $X_{2} \subset g$ and $Y_{2}-Y_{1} \subset g^{\prime}$ their sum cannot be a point of $g$ unless $Y_{2}-Y_{1}=O$. But then $Y_{2}=Y_{1}$ and $X_{2}=X_{1}$.

VI. By virtue of $\mathrm{V}$ there may be uniquely associated with every point $Z=X+Y\left(X \subset g, Y \subset g^{\prime}\right)$ of $p$ the isometric coordinates $x, y$ of $X$ and $Y$ respectively. Since, conversely, to every coordinate-pair $(x, y)$ there exists uniquely a corresponding point $Z \subset p$, a one-to-one mapping may thus be set up between $p$ and the euclidean plane $e$. 
It follows from statement $4, \S 2$, and IV that this mapping is a homeomorphism.

VII. The S.L. of $p$ are mapped into the S.L. of e. ${ }^{5}$ For, by (1), we may write

$$
\begin{aligned}
M\left(Z_{1}, Z_{2}\right) & =\left(X_{1}+Y_{1}\right) / 2+\left(X_{2}+Y_{2}\right) / 2 \\
& =\left(X_{1}+X_{2}\right) / 2+\left(Y_{1}+Y_{2}\right) / 2, \\
E_{1} & =E\left(Z_{1}, Z_{2}\right)=2\left(X_{1}+Y_{1}\right)-\left(X_{2}+Y_{2}\right) \\
& =\left(2 X_{1}-X_{2}\right)+\left(2 Y_{1}-Y_{2}\right), \\
E_{2} & =\left(Z_{1}, Z_{2}\right) E=2\left(X_{2}+Y_{2}\right)-\left(X_{1}+Y_{1}\right) \\
& =\left(2 X_{2}-X_{1}\right)+\left(2 Y_{2}-Y_{1}\right),
\end{aligned}
$$

so that the coordinates in $e$ corresponding to $M, E_{1}, E_{2}$ are respectively $\left(x_{1} / 2+x_{2} / 2, y_{1} / 2+y_{2} / 2\right),\left(2 x_{1}-x_{2}, 2 y_{1}-y_{2}\right),\left(2 x_{2}-x_{1}, 2 y_{2}-y_{1}\right)$. Thus the centers of any pair of points in $R$ map into the corresponding $e$-centers. The statement is then proved by repeatedly taking centers and their limit points, ${ }^{6}$ and using the fact that the mapping is a homeomorphism. A consequence of this and the fact that the whole of $e$ is covered by the mapping is that the $e$ parallel axiom holds.

VIII. $p$ admits of a simply-transitive abelian group of translations. We shall prove that the translations

$$
P:(x, y) \rightarrow P^{T}:(x+a, y+b), \quad T=T_{a b},
$$

of the euclidean plane are motions for the given metric. It is well known and very easy to prove that the metric of $p$ is then Minkowskian. ${ }^{7}$

1. If, for a set of points $P_{0}, P_{1}, \cdots, P_{n}, \cdots$ on an S.L. $g \subset p$ we have $P_{i-1} P_{i}=P_{i} P_{i+1}$, then $P_{i-1}^{T} P_{i}^{T}=P_{i}^{T} P_{i+1}^{T}, i=1, \cdots, n, \cdots$ For we have $P_{i}=M\left(P_{i-1} P_{i+1}\right)$, which, by VII, has as image in $e$ the corresponding $e$-center. Since this, in turn, remains center under the mapping (2), and then under the inverse mapping of $e$ into $p$, the statement is proved.

2. If $P^{T}, P^{T^{2}}, \cdots, P^{T^{n}}, \cdots$ are the images of $P$ under $T, T^{2}, \cdots$, $T^{n}, \cdots$ then $P P^{T}=P^{T^{n}} P^{T^{n+1}}, n=0,1, \cdots$. For let $P \rightarrow(x, y)$, then $P^{T^{n}} \rightarrow(x+n a, y+n b)$, and since this point is the $e$-center of $(x+(n-1) a, y+(n-1) b)$ and $(x+(n+1) a, y+(n+1) b), P^{T^{n}}$ must be the center of $P^{T^{n-1}}$ and $P^{T^{n+1}}$, that is, $P^{T^{n-1}} P^{T^{n}}=P^{T^{n}} P^{T^{n+1}}$, $n=0,1, \cdots$.

${ }^{5}$ When such a mapping is possible we say that the metric of $p$ is Desarguesian.

- This process is exemplified in [1, p. 87].

${ }^{7}$ See $[2$, Theorem II, 1.17a] (invariance under translation is equivalent to dependence on $x-y$ ). 
3. If, for a set of points $P_{0}, P_{1}, \cdots, P_{n}, \cdots$, on an S.L. $g \subset p$, $P_{i-1} P_{i}=P_{i} P_{i+1}$, then $P_{0}, P_{1}{ }^{T}, P_{2} T^{2}, \ldots, P_{n}^{T^{n}}$ lie on an S.L. and $P_{0} P_{i}^{T}=P_{i-1}^{T i-1} P_{i}^{T^{i}}, i=1, \cdots, n, \cdots$. This follows from the fact that the corresponding relations hold in $e$.

4. We shall now prove that if $P, Q \subset p$ then $P Q=P^{T} Q^{T}$. Set $P=P_{0}$, $Q=P_{1}$ in the notation of the three previous steps. Then $n P Q=P_{0} P_{n}$ $\leqq P_{0} P_{0}^{T}+P_{0}^{T} P_{n}^{T}+P_{n}^{T} P_{n}$. But by step $1, P_{0}^{T} P_{n}^{T}=n P^{T} Q^{T}$ and by step two $P_{n} P_{n}^{T}=P_{n} P_{n}^{T^{n}} / n$, hence $n\left|P Q-P^{T} Q^{T}\right| \leqq P_{n} P_{n}^{T^{n}} / n+P_{0} P_{0}^{T} \leqq P_{0} P_{1}$ $+P_{0} P_{1}^{T}+P_{0} P_{0}^{T}$, since by step $3, \quad P_{n} P_{n}^{T n} \leqq P_{0} P_{n}+P_{0} P_{n}^{T^{n}}=n P_{0} P_{1}$ $+n P_{0} P_{1}^{T}$. Since $n$ can be made arbitrarily large, $P Q=P^{T} Q^{T}$. Thus $T$ is proved to be a congruence, and since there are no fixed points, it is a translation. As the translations of $e$ are simply-transitive and abelian, so are those of $p$.

This completes the proof that any three points $O, X, Y$ of $R$ are contained in a Minkowski plane. Note that we have proved that this is a consequence of associative segment addition merely with respect to a single point as origin. From the existence of translations it follows that addition is associative with respect to every point. The main theorem then follows immediately for S.L. spaces of dimension not less than 2. For the space is Desarguesian (see [2, Theorem III, 6.3]), and, using the same methods as above, the transitive Abelian group of the form $X \rightarrow X+h$ can be introduced. That these transformations are motions follows immediately from the fact that $X, Y, X \rightarrow h$, $Y \rightarrow h$ lie in a plane, hence $X Y=(X+h)(Y+h)$. The finite-compactness of the space together with its linear operation imply its finitedimensionality.

Thanks are due to H. Busemann, K. Menger, and his associates for helpful suggestions and criticisms.

\section{REFERENCES}

1. K. Menger, Untersuchungen über allgemeine Metrik, I, II, III. Math. Ann. vol. 100 (1928) pp. 75-163.

2. H. Busemann, Metric methods in Finsler spaces and in the foundations of geometry, Annals of Mathematics Studies, no. 8, Princeton, 1942. 519.

3. A. A. Albert, Quasigroups. I, Trans. Amer. Math. Soc. vol. 54 (1943) pp. 507-

Johns Hopkins University 